Connect and Strive to Survive and Thrive:

The Evolutionary Meaning of Communion and Agency

Todd Chan, Iris Wang, \& Oscar Ybarra

Department of Psychology, University of Michigan

530 Church St, Ann Arbor, MI, 48109-1043

\title{
Citation:
}

Chan, T., Wang, I., \& Ybarra, O. (2018). Connect and strive to survive and thrive: The evolutionary meaning of communion and agency. In Abele, A. \& B. Wojciszke (Eds.) Agency and Communion in Social Psychology. 
Connect and Strive to Survive and Thrive:

The Evolutionary Meaning of Communion and Agency

Humans have a universal drive to understand other people's intentions and behavior. From an evolutionary perspective, being perceptive about others in the social world-especially strangers — comes with a host of benefits that promote survival (Cosmides \& Tooby, 1992). People need to decide whether a person poses a threat or an opportunity, and quickly and accurately enough. Fortunately, humans have a well-developed, parsimonious method for perceiving others, where individuals and situations that we encounter are quickly evaluated under two dimensions, known as communion and agency.

In this chapter, we start by defining what these two fundamental dimensions refer to in the context of person evaluation, and we provide evidence that people, across culture and contexts, readily evaluate people with this communion-agency lens. Then, we explain how these two dimensions provide functional benefits in how effectively people can (a) connect with others and (b) reach one's goals - two core human motivations related to survival. Finally, we illustrate how knowing how communal and agentic other people are can confer specific benefits for solving three recurring evolutionary challenges: acquiring status, long-term mating, and reproducing.

\section{The Big Two}

Perceptual and cognitive systems evolved to address recurrent problems that humans have faced throughout their evolutionary history. People in group life, across time and culture, have needed to fulfill two broad motives: to engage with others and to pursue personal goals and 
distinctiveness, and in that order (Ybarra et al., 2008). Social perception appears to be organized around these two evolutionary motives of belonging and individual goal-pursuit. Consider when we meet a new person, deciding whether someone is a friend or foe relies on knowing (a) what this person's intentions are, and (b) how these intentions facilitate or hinder our survival, belonging, and goal-pursuit. To answer these questions, people benefit from knowing whether people are worthy of connecting (i.e., communal) and whether they are competent (i.e., agentic).

Termed the "Big Two,"1 the dimensions of communion and agency account in large measure for how people think about others (Bakan, 1966). Communion refers to individuals' pro-sociality, affiliative tendencies, and kindness. For example, a highly communal individual may be described as someone who is fair, good-natured, honest, loyal, selfless, sincere, and truthful (Wojciszke, Baryla, Parzuchowski, Szymkow, \& Abele, 2011). Agency refers to individuals' abilities, skills, and ambitions. A highly agentic individual may likewise be described as someone who is clever, competent, efficient, energetic, intelligent, knowledgeable, and logical (Wojciszke et al., 2011). Thus, communion refers to how well individuals connect with other people, whereas agency refers to how well individuals can achieve goals.

These two dimensions are orthogonal but can interact to produce different impressions (Kervyn, Yzerbyt, \& Judd, 2010). This interaction is key from an evolutionary perspective. For example, someone who is categorized as low on communion may be perceived as untrustworthy, yet whether they are seen as a threat to an individual or group depends on whether they are also perceived to be sufficiently agentic (i.e., competent) to pursue any malicious intentions (Fiske, Cuddy, \& Glick, 2007). As we will discuss, how someone is perceived and treated often depends

\footnotetext{
${ }^{1}$ Different lines of research have termed these dimensions differently (e.g., warmth and competence; dominance and warmth) (see Abele \& Wojciszke, 2014).
} 
on the perception generated by the interaction of these dimensions (i.e., opportunity or threat) and the goals of the perceiver.

Much research shows that when people generate descriptions and judgments of themselves, other individuals, or groups, they rely on these dimensions of communion and agency. For example, when people are asked to describe themselves or other people in their own words, between $75 \%$ and $85 \%$ of their open responses contain descriptions that fall under the dimensions of communion or agency (Abele \& Bruckmüller, 2011; 2013; Uchronski, 2008). Across cultures, whether individuals or groups are concerned, impressions of their communion and agency largely account for the variance in global perceptions of them (Cuddy et al., 2009; Kervyn, Yzerbyt, Demoulin, \& Judd, 2008; Phalet \& Poppe, 1997; Wojciszke, Bazinska, \& Jaworski, 1998). This innate preparedness to structure and see the world through the lens of communion and agency suggests doing so helps meet fundamental needs and was functional in solving recurring evolutionary challenges. We now turn to discussing how these dimensions of communion and agency can respectively help people connect and strive.

\section{Communion for Connecting}

To belong to a group and to be accepted by others is a fundamental human need. From an evolutionary perspective, social exclusion meant lack of resources and no protection from threats, and thus, inevitable death (Baumeister \& Leary, 1995). Conversely, belonging to a group confers direct benefits for oneself in the way of protection, reducing threats, and the sharing of skills and resources (West, Griffin, \& Gardner, 2007). Within groups, selection likely favored affiliating with cooperative, agreeable people who contribute, and avoiding freeloading, cheating people who deplete from group life (Neuberg \& Cottrell, 2008). Indeed, research shows that in 
social dilemmas, socially agreeable individuals are more likely to change their individual behaviors to favor what would be best for the group, suggesting there is a strong basis to assume that highly communal people confer direct benefits for group life (Koole, Jager, van den Berg, Vlek, \& Hofstee, 2001). Thus, people are acutely attuned to whether other people show traits that signal that they are affiliative. Individuals need to be assured that others are cooperative enough to provide these benefits (vs. confer harm or threats to them), and that when they help others, others can be trusted to return the favor (i.e., reciprocal altruism) (Trivers, 1971).

How do communal evaluations inform these decisions about affiliation, trust, and cooperation? First, communal traits reflect how affiliative others are. Second, they signal whether a person is likely to violate the rules of group life. Third, they provide cues of resource value, or the likelihood that a person will share a resource, cooperate, and reciprocate (Scholer \& Higgins, 2008). Knowing someone is immoral, hostile, or disagreeable - negative communal qualities - are salient threat cues that they will be un-affiliative, violate the rules of social life, and be of low resource value. Conversely, positive communal evaluations (e.g., honest and moral) reduce perceptions of threat, resulting in a more positive evaluation overall (Brambilla, Sacchi, Rusconi, Cherubini, \& Yzerbyt, 2011). Thus, communal traits broadly inform whether an interaction will be a cooperative or threatening one.

\section{Detect Cooperation and Amplify Threat}

Several features of communal evaluations support the notion that they serve the purpose of detecting cooperation and amplifying threat. 
First, people are quite adept at automatically judging how trustworthy, agreeable, cooperative, honest, and extraverted others are from only facial cues (Stirrat \& Perrett, 2010; Todorov, Baron, \& Oosterhof, 2008; Zebrowitz, 2017).

Second, people are highly interested in ensuring others they interact with are communal. For example, when people are asked to describe an ideal group member, people tend to prioritize communal traits (e.g., trustworthiness) over agentic traits (e.g., intelligence) regardless of group context (e.g., work or family member) (Cottrell, Neuberg, \& Li, 2007). Likewise, when individuals are asked what information they would like to know about another person, they report being more interested in learning about communal features of the person over agentic ones (De Bruin \& van Lange, 2000; Wojciszke et al., 1998). Even when toddlers are asked to evaluate whether claims provided by two informants are true, they tend to trust the knowledge of the “nicer” individual (Landrum, Mills, \& Johnston, 2013).

Third, there is an asymmetrical sensitivity in communal evaluations towards negative information, and negative information is strongly remembered. For example, although people are adept at detecting whom to trust, people are even more sensitive at detecting those who violate the rules. After being exposed to faces of individuals identified as cheaters or as trustworthy individuals, people were more likely to remember the cheaters' faces a week later (Mealey, Daood, \& Krage, 1996).

This sensitivity to negative communion evaluations may stem from evolutionary mechanisms designed to err on the side of caution when assessing and reducing threats (i.e., error management theory) (Nesse, 2005). That is, the consequences of misjudging someone as uncooperative (when they are cooperative) is likely to be less costly, harmful, or lethal than the consequences of misjudging someone as cooperative (when they are not). Thus, when cues like 
cooperation are difficult to detect accurately, it is often better to err on the evaluation of less consequence when choosing to enter social interactions. Consistent with this notion, when initial communal evaluations are negative, they are resistant to revision even in light of future information or behavior (Reeder \& Brewer, 1979; Ybarra, Chan, \& Park, 2001). For example, after being told someone is hostile, all future behavior from that individual is likely to be filtered through this "hostile" label, coloring even subsequently positive behavioral information. However, when initial communal evaluations are positive, only one instance of negative information is necessary to irreversibly update one's global evaluation in a negative direction (Reeder \& Brewer, 1979; Skowronski \& Carlston, 1989; Ybarra et al., 2001).

In sum, relative to other information obtained from evaluations, communal information is primary, spontaneous, salient, and well-remembered. These features serve the function of detecting whom to affiliate with and whom to avoid or treat with caution. Knowing this information helps individuals avoid threats to survival, and to select groups and others who can be relied on in order to meet one's own goals.

\section{Agency for Striving}

Once the problem of positively connecting with others is sufficiently solved, people must turn their attention to a second need: pursuing individual distinction and personal goals (Ybarra et al., 2008). People who acquire unique skills acquire more resources, mating partners, and status and respect from the group (Gebauer, Sedikides, Lüdtke, \& Neberich, 2014; Schmitt \& Buss, 1996). Similarly, people's social reputation is comprised of how similar they are to others (i.e., being able to get along; communion) but also by how different they are from others (i.e., 
being able to get ahead; agency) (Leary, 2007). Thus, people who do not strive to get ahead get left behind.

Critically, pursuing goals and getting ahead nonetheless relies heavily on the contributions of other people. Whether it be hunting, building, or using language, most human practices are developed by learning, modeling, and assistance from other people (Tomasello, 1998). How do we identify who to learn from? We now turn our attention to the agency dimension, which helps answer this question (Peeters \& Czapinski, 1990). Of course, given that these evaluations still involve others, and consistent with the preeminence and dominance of communion in people's evaluations, our discussion of agency is intertwined with the fact that agentic evaluations invariably interact with communal evaluations to provide an informative impression.

Evaluating others on agency is concerned with assessing how others provide value (or lack thereof) for one's own goals. Specifically, being able to correctly identify those to imitate, those who will share skills or knowledge, and those who will provide favors or direct assistance, confers benefits when striving to get ahead. Threats can not only arise from affiliating with those who are un-communal, but also those who are un-agentic. For example, investing effort in imitating those who are incompetent may lead to wasted time or stifled skill development.

Further, people need to identify those who will interfere with their goal pursuit and identify those who are competitors when resources are limited or obtained through competition. For example, if someone is evaluated relative to oneself as highly agentic, they are likely to have increased influence over other people and resources. Should this person also be highly communal, they may be seen as an admired teacher that one should seek out for access, assistance, or modeling in order to reach one's own goals. In contrast, should this person not be 
very communal, they may be seen as an unhelpful competitor one should avoid wasting time on when seeking assistance but instead be vigilant of in competition (c.f. Cuddy, Glick, \& Beninger, 2011). In sum, knowing who is agentic can aid individuals' goal pursuit.

Consistent with the notion that agency serves functions related to individual goal pursuit, how interested people are in processing agentic information depends on how relevant the situation is to their goal-pursuit (Abele \& Wojciszke, 2014). For example, whether meeting a stranger or a doctor, people should want to deal with someone who is kind, polite, and caring. Thus, people are concerned with communal evaluations as a default. However, relative to a stranger, people should place more weight on evaluating their doctor on an agentic dimension, given that a doctor is more important for one's personal goals (i.e., maintaining health). When it comes to evaluating others on agency, context plays more of a role in how people approach and process information from this dimension.

Similarly, the goals and skills people concern themselves with will vary depending on environment and context. For example, individuals living in the tundra require different skills than those living in the desert. Likewise, what is considered to be a coveted goal, or what types of behaviors or traits confer respect with one's peers, are likely to vary among different cultures. Accordingly, whereas people tend to highly agree on what traits and behaviors are communal, what constitutes agentic traits, behaviors, and practices are more likely to vary depending on the specific environment and culture individuals find themselves in and the opportunities afforded to them (Ybarra et al., 2008).

Given the relevance of agency to goal pursuit, in contrast to communion, a focus on processing and weighting positive over negative information is likely to confer advantages for individuals seeking assistance in meeting their goals. That is, the costs of identifying someone as 
un-agentic when they are, is likely to be more costly than identifying someone as agentic when they are not. Misidentifying someone as un-agentic (when they are) may lead to missed opportunities to learn or missing out on resources due to underestimating one's competitors; conversely, the costs of misidentifying someone as agentic (when they are not) may be wasting time working with someone who turns out to be of little assistance or needlessly exerting energy competing with someone who turns out not to be a competitor. Further, because it is relatively difficult to "fake" competence, instances of competence are more likely to be true indicators of positive agency. In contrast, instances of failure can be attributed to various idiosyncratic reasons, and without additional evidence, may lead to misidentifying someone as un-agentic when they in fact are (Skowronski \& Carlston, 1989). Thus, it is likely more costly to prematurely dismiss someone as ineffective when they are not than to work with someone who turns out to be so.

Indeed, research shows that agentic evaluations show an asymmetrical sensitivity, but in this case to positive information (Reeder \& Brewer, 1979; Skowronski \& Carlston, 1989). When people are in competitive contexts, for example, they report wanting to know more about the positive strengths and qualities of their opponent over their weaknesses, and remember this positive agentic information better (Chan \& Ybarra, 2002). Thus, in their overall evaluations of agency, people are more likely to weight information that suggests another person is able and competent over information that shows that they are failing or incompetent.

\section{Solving Specific Challenges}

We have discussed how leveraging information gleaned from evaluating others on communion and agency provides benefits for connecting and striving. We now turn to 
illustrating how evaluations of communion and agency, together, serve to help people address three core evolutionary challenges: gaining status, long-term mating, and parenting (Ackerman, Huang, \& Bargh, 2012).

\section{Gaining Status}

Increasing one's status is one universal and core challenge that humans continuously strive to solve (Anderson, Hildreth, \& Howland, 2015). Essentially, obtaining status is a relative goal, a goal to increase one's standing, importance, and recognition in the social hierarchy (Fiske, 2010). Increasing one's status brings a host of benefits, such as increased access to resources, favors, esteem from others, and mates (de Waal \& de Waal, 2007; van Vugt \& Tybur, 2015). Status can be acquired by exerting physical dominance over others, demonstrating that one has relatively unique skills, resources, or information, or showing that one is particularly moral or virtuous (Bai, 2016; Kenrick, Griskevicius, Neuberg, \& Schaller, 2010). Again, what skills and information are valued in a social context depend on the ecology in which people live, which dictate what skills are advantageous for problem solving. Nonetheless, given that status is inherently imbued with agentic characteristics (being competent, unique, influential, and powerful), people who want to attain status should demonstrate agentic behaviors (cf. Cheng, Tracy, \& Henrich, 2010).

For people seeking status, evaluations of agency and communion are critical. First, given that increasing status inherently depends on others' relative standing, people first need to acquire accurate information about the social hierarchy, which they appear very motivated to do (Moors \& De Houwer, 2005). Given the tight link between agency and status, having evaluations of others' agency is necessary to situate one's own status among those who are higher and lower 
than oneself on the hierarchy (de Waal, 1986). Second, after knowing the status hierarchy, people can leverage this information to "get ahead" by identifying those on the hierarchy whom they can affiliate with, model, or seek assistance from in developing skills that can increase their own status. Individuals with high-status receive benefits and resources from transferring their knowledge to others (van Vugt \& Tybur, 2015). Of course, whether someone is an eager teacher will also depend on how communal that person is, or how willing that person is to share knowledge and resources, consistent with the proposal that agentic traits usually interact with communal ones (Cuddy et al., 2011).

At the same time, people can use this information to identify who their competitors are, and whether they should compete with these individuals or avoid them (Cuddy, Fiske, \& Glick, 2008). If the higher-status individual is one who is competing for the same resource or pursuing the same goal, the person may pose a threat that may be best dealt with by avoiding this individual; if this higher-status person is pursuing a different goal, the person may be a potential mentor. Alternatively, if it is known that a lower-status individual is the person who is competing for the same resource, people may leverage this information to compete more fiercely in order to protect their own status. In sum, deftly recognizing agency and communion, with their links to striving and connecting, directly informs how individuals may most adaptively navigating this universal challenge of increasing one's status.

\section{Long-Term Mating \& Reproduction}

Beyond one's own survival and growth, people strive to have offspring that live long enough to reproduce themselves (Ackerman et al., 2012). In order to reproduce and nurture offspring effectively, however, people need to seek out a long-term mating partner who will also 
act as a parent. With regards to long-term mating then, person evaluation inherently involves evaluating their suitability as a parent (Miller \& Todd, 1998). Given the long-term nature of parenthood, individuals are motivated to find someone who shows fidelity and investment in the relationship (Buss \& Schmitt, 1993; Li, Balley, Kenrick, \& Linsenmeier, 2002). However, simply being committed to the relationship is insufficient; people, particularly females, are also motivated to seek out mates who are competent enough to acquire resources and nurture offspring.

How can evaluations of communion and agency inform whether someone meets these criteria? Communal attributes - being kind, gregarious, and sociable - are associated with increased cooperation, loyalty, and fidelity in relationships, and as such, individuals searching for long-term relationships tend to desire a partner who is, first and foremost, highly communal (Buss \& Schmitt, 1993; Li et al., 2002; Nettle \& Clegg, 2008). These traits also help inform whether individuals will invest in their dependent offspring. Communal traits signal that an individual possesses the nurturing qualities that are critical for effective parenting. Women tend to be viewed as more communal than agentic, perhaps reflective of the fact that they are the sex that provides greater investment in the nurturing of children (L. B. Lueptow, Garovich, \& Lueptow, 1995; Williams \& Best, 1982).

However, people also report a strong desire for an agentic partner. In fact, of the Big Five, after agreeableness (i.e., communion), openness to experience, linked with creativity and intelligence (Moutafi, Furnham, \& Crump, 2006), is the most valued factor in a mate (Botwin, Buss, \& Shackelford, 1997). Both males and females report desiring a partner that is open, curious, interesting, and intellectual, all agency related attributes, suggesting that these qualities signal fitness in a partner (Buss, 1989). For example, these traits show that an individual 
possesses the capability to protect their offspring, acquire resources, and pass on skills and knowledge.

Although agentic information is valued by both sexes, it appears that females are more likely to privilege evaluations on this dimension (e.g., of intelligence, resources, status) in the context of long-term mating (Buss, 1989). Females put forth greater investment in the bearing and rearing of a child (Trivers, 1972). As such, they are more oriented towards choosing a mate that can acquire and invest sustained resources in themselves and their offspring (Buss, 1989).

At the same time, like status, males also need to know other males' level of agency to effectively pursue their goal of attracting and retaining a mate. Given that agentic, high-status individuals (especially males) attract more mates, to effectively engage and succeed (get ahead) at intra-sexual competition requires being aware of the relative success, status, and resources of one's competitors (Cummins, 2005). In sum, using agency to supplement evaluations of communion when evaluating individuals as mating partners can provide a wealth of information that can facilitate the process of finding and attracting a suitable long-term mate as well as partner in parenting.

\section{Conclusion}

Over human evolutionary history, humans have had two fundamental motivations: connect with others and develop personal skills and goals. Neither of these motivations can be accomplished alone, however, and those who succeed at meeting these motivations know whom to leverage for assistance and whom to avoid as a threat. Evaluating others on how communal and agentic they are serves to parsimoniously inform this question. In particular, as illustrated in this chapter, they can confer direct benefits for how people can efficiently acquire status and 
evaluate people for their long-term potential as mates and as parents. Ultimately, these two dimensions serve to guide how humans process information in the way that is most adaptive to their surviving and thriving. 


\section{References}

Abele, A. E., \& Bruckmüller, S. (2011). The bigger one of the "Big Two?" Preferential processing of communal information. Journal of Experimental Social Psychology, 47(5), 935-948.

Abele, A. E., \& Bruckmüller, S. (2013). The big two of agency and communion in language and communication. Social Cognition and Communication, 173-184.

Abele, A. E., \& Wojciszke, B. (2014). Communal and agentic content in social cognition: A dual perspective model. Advances in Experimental Social Psychology, 50, 195-255.

Ackerman, J. M., Huang, J. Y., \& Bargh, J. A. (2012). Evolutionary perspectives on social cognition. The Handbook of Social Cognition, 451-473.

Anderson, C., Hildreth, J. A. D., \& Howland, L. (2015). Is the desire for status a fundamental human motive? A review of the empirical literature. Psychological Bulletin, 141(3), 574601.

Bai, F. (2016). Beyond dominance and competence: A moral virtue theory of status attainment. Personality and Social Psychology Review, 21(3), 203-227.

Bakan, D. (1966). The duality of human existence: An essay on psychology and religion.

Baumeister, R. F., \& Leary, M. R. (1995). The need to belong: Desire for interpersonal attachments as a fundamental human motivation. Psychological Bulletin, 117(3), 497-529.

Botwin, M. D., Buss, D. M., \& Shackelford, T. K. (1997). Personality and mate preferences: Five factors in mate selection and marital satisfaction. Journal of Personality, 65(1), 107136.

Brambilla, M., Sacchi, S., Rusconi, P., Cherubini, P., \& Yzerbyt, V. Y. (2011). You want to give a good impression? Be honest! Moral traits dominate group impression formation. British Journal of Social Psychology, 51(1), 149-166.

Buss, D. M. (1989). Sex differences in human mate preferences: Evolutionary hypotheses tested in 37 cultures. Behavioral and Brain Sciences, 12(1), 1-14.

Buss, D. M., \& Schmitt, D. P. (1993). Sexual strategies theory: an evolutionary perspective on human mating. Psychological Review, 100(2), 204-232.

Chan, E., \& Ybarra, O. (2002). Interaction goals and social information processing: Underestimating one"s partners but overestimating one"s opponents. Social Cognition, 20(5), 409-439.

Cheng, J. T., Tracy, J. L., \& Henrich, J. (2010). Pride, personality, and the evolutionary foundations of human social status. Evolution and Human Behavior, 31(5), 334-347.

Cosmides, L., \& Tooby, J. (1992). Cognitive Adaptations for Social Exchange. In J. Barkow, L. Cosmides, \& J. Tooby (Eds.), The Adapted Mind: Evolutionary Psychology and the Generation of Culture (pp. 163-228). The adapted mind.

Cottrell, C. A., Neuberg, S. L., \& Li, N. P. (2007). What do people desire in others? A sociofunctional perspective on the importance of different valued characteristics. Journal of Personality and Social Psychology, 92(2), 208.

Cuddy, A. J., Fiske, S. T., \& Glick, P. (2008). Warmth and competence as universal dimensions of social perception: The stereotype content model and the BIAS map. Advances in Experimental Social Psychology, 40, 61-149.

Cuddy, A. J., Fiske, S. T., Kwan, V. S., Glick, P., Demoulin, S., Leyens, J. P., et al. (2009). 
Stereotype content model across cultures: Towards universal similarities and some differences. British Journal of Social Psychology, 48(1), 1-33.

Cuddy, A. J., Glick, P., \& Beninger, A. (2011). The dynamics of warmth and competence judgments, and their outcomes in organizations. Research in Organizational Behavior, 31, 73-98.

Cummins, D. (2005). Dominance, status, and social hierarchies. In D. M. Buss (Ed.), The Handbook of Evolutionary Psychology (pp. 676-697). Hoboken, NJ: Wiley.

De Bruin, E. N. M., \& van Lange, P. A. M. (2000). What people look for in others: Influences of the perceiver and the perceived on information selection. Personality and Social Psychology Bulletin, 26(2), 206-219.

de Waal, F. B. (1986). The integration of dominance and social bonding in primates. The Quarterly Review of Biology, 61(4), 459-479.

de Waal, F., \& de Waal, F. B. (2007). Chimpanzee politics: Sex and power among apes. Johns Hopkins University Press.

Fiske, S. T. (2010). Interpersonal stratification: Status, power, and subordination. In S. T. Fiske, D. T. Gilbert, \& G. Lindzey (Eds.), (pp. 941-982). Hoboken, NJ: Wiley.

Fiske, S. T., Cuddy, A. J., \& Glick, P. (2007). Universal dimensions of social cognition: Warmth and competence. Trends in Cognitive Sciences, 11(2), 77-83.

Gebauer, J. E., Sedikides, C., Lüdtke, O., \& Neberich, W. (2014). Agency-communion and interest in prosocial behavior: Social motives for assimilation and contrast explain sociocultural inconsistencies. Journal of Personality, 82(5), 452-466.

Kenrick, D. T., Griskevicius, V., Neuberg, S. L., \& Schaller, M. (2010). Renovating the pyramid of needs: Contemporary extensions built upon ancient foundations. Perspectives on Psychological Science, 5(3), 292-314.

Kervyn, N., Yzerbyt, V. Y., Demoulin, S., \& Judd, C. M. (2008). Competence and warmth in context: The compensatory nature of stereotypic views of national groups. European Journal of Social Psychology, 38(7), 1175-1183.

Kervyn, N., Yzerbyt, V., \& Judd, C. M. (2010). Compensation between warmth and competence: Antecedents and consequences of a negative relation between the two fundamental dimensions of social perception. European Review of Social Psychology, 21(1), 155-187.

Koole, S. L., Jager, W., van den Berg, A. E., Vlek, C. A. J., \& Hofstee, W. K. B. (2001). On the social nature of personality: Effects of extraversion, agreeableness, and feedback about collective resource use on cooperation in a resource dilemma. Personality and Social Psychology Bulletin, 27(3), 289-301.

Landrum, A. R., Mills, C. M., \& Johnston, A. M. (2013). When do children trust the expert? Benevolence information influences children's trust more than expertise. Developmental Science, 16(4), 622-638.

Leary, M. R. (2007). The curse of the self: Self-awareness, egotism, and the quality of human life. New York: Oxford University Press.

Li, N. P., Balley, J. M., Kenrick, D. T., \& Linsenmeier, J. A. (2002). The necessities and luxuries of mate preferences: Testing the tradeoffs. Journal of Personality and Social Psychology, 82(6), 947-955.

Lueptow, L. B., Garovich, L., \& Lueptow, M. B. (1995). The persistence of gender stereotypes in the face of changing sex roles: Evidence contrary to the sociocultural model. Ethology and Sociobiology, 16(6), 509-530.

Mealey, L., Daood, C., \& Krage, M. (1996). Enhanced memory for faces of cheaters. Ethology 
and Sociobiology, 17(2), 119-128.

Miller, G. F., \& Todd, P. M. (1998). Mate choice turns cognitive. Trends in Cognitive Sciences, 2(5), 190-198.

Moors, A., \& De Houwer, J. (2005). Automatic processing of dominance and submissiveness. Experimental Psychology, 52(4), 296-302.

Moutafi, J., Furnham, A., \& Crump, J. (2006). What facets of openness and conscientiousness predict fluid intelligence score? Learning and Individual Differences, 16(1), 31-42.

Nesse, R. M. (2005). Natural selection and the regulation of defenses: A signal detection analysis of the smoke detector principle. Evolution and Human Behavior, 26(1), 88-105.

Nettle, D., \& Clegg, H. (2008). Personality, mating strategies, and mating intelligence. In G. Geher \& G. Miller (Eds.), Mating Intelligence; Sex, Relationships, and the Minds Reproductive System (pp. 121-135). Lawrence Erlbaum.

Neuberg, S. L., \& Cottrell, C. A. (2008). Managing the threats and opportunities afforded by human sociality. Group Dynamics: Theory, Research, and Practice, 12(1), 63-72.

Peeters, G., \& Czapinski, J. (1990). Positive-negative asymmetry in evaluations: The distinction between affective and informational negativity effects. European Review of Social Psychology, 1(1), 33-60.

Phalet, K., \& Poppe, E. (1997). Competence and morality dimensions of national and ethnic stereotypes: A study in six eastern-European countries. European Journal of Social Psychology, 27(6), 703-723.

Reeder, G. D., \& Brewer, M. B. (1979). A schematic model of dispositional attribution in interpersonal perception. Psychological Review, 86(1), 61.

Schmitt, D. P., \& Buss, D. M. (1996). Strategic self-promotion and competitor derogation: Sex and context effects on the perceived effectiveness of mate attraction tactics. Journal of Personality and Social Psychology, 70(6), 1185-1204.

Scholer, A. A., \& Higgins, E. T. (2008). Distinguishing levels of approach and avoidance: An analysis using regulatory focus theory. In Handbook of Approach and Avoidance Motivation (pp. 489-503). New York, NY, US: Psychology Press.

Skowronski, J. J., \& Carlston, D. E. (1989). Negativity and extremity biases in impression formation: A review of explanations. Psychological Bulletin, 105(1), 131.

Stirrat, M., \& Perrett, D. I. (2010). Valid facial cues to cooperation and trust: Male facial width and trustworthiness. Psychological Science, 21(3), 349-354.

Todorov, A., Baron, S. G., \& Oosterhof, N. N. (2008). Evaluating face trustworthiness: a model based approach. Social Cognitive and Affective Neuroscience, 3(2), 119-127.

Tomasello, M. (1998). Emulation learning and cultural learning. Behavioral and Brain Sciences, 21(5), 703-704.

Trivers, R. (1972). Parental investment and sexual selection. In B. G. Campbell (Ed.), Sexual Selection and the Descent of Man (pp. 136-179). Chicago: Aldine Publishing Company.

Trivers, R. L. (1971). The evolution of reciprocal altruism. The Quarterly Review of Biology, 46(1), 35-57.

Uchronski, M. (2008). Agency and communion in spontaneous self-descriptions: Occurrence and situational malleability. European Journal of Social Psychology, 38(7), 1093-1102.

van Vugt, M., \& Tybur, J. M. (2015). The evolutionary foundations of status hierarchy. In D. M. Buss (Ed.), (pp. 788-809). Hoboken, NJ: Wiley.

West, S. A., Griffin, A. S., \& Gardner, A. (2007). Evolutionary explanations for cooperation. Current Biology, 17(16), R661-R672. 
Williams, J. E., \& Best, D. L. (1982). Measuring sex stereotypes: A thirty-nation study. Newbury Park, CA: Sage.

Wojciszke, B., Baryla, W., Parzuchowski, M., Szymkow, A., \& Abele, A. E. (2011). Self-esteem is dominated by agentic over communal information. European Journal of Social Psychology, 41(5), 617-627.

Wojciszke, B., Bazinska, R., \& Jaworski, M. (1998). On the dominance of moral categories in impression formation. Personality and Social Psychology Bulletin, 24(12), 1251-1263.

Ybarra, O., Chan, E., \& Park, D. (2001). Young and Old Adults' Concerns About Morality and Competence. Motivation and Emotion, 25(2), 85-100.

Ybarra, O., Chan, E., Park, H., Burnstein, E., Monin, B., \& Stanik, C. (2008). Life's recurring challenges and the fundamental dimensions: An integration and its implications for cultural differences and similarities. European Journal of Social Psychology, 38(7), 1083-1092.

Zebrowitz, L. A. (2017). First impressions from faces. Current Directions in Psychological Science, 26(3), 237-242. 